\title{
Research Article On $\mathrm{FS}_{+}$-Domains
}

\author{
Yayan Yuan and Jibo Li \\ College of Mathematics and Information Science, Henan Normal University, Xinxiang, Henan 453007, China \\ Correspondence should be addressed to Yayan Yuan; yayanyuan@hotmail.com
}

Received 20 January 2014; Accepted 24 February 2014; Published 14 May 2014

Academic Editor: Changsen Yang

Copyright (C) 2014 Y. Yuan and J. Li. This is an open access article distributed under the Creative Commons Attribution License, which permits unrestricted use, distribution, and reproduction in any medium, provided the original work is properly cited.

\begin{abstract}
We introduce a new construction-FS - -domain - and prove that the category with $F S_{+}$-domains as objects and Scott continuous functions as morphisms is a Cartesian closed category. We obtain that the Plotkin powerdomain $P^{P}(L)$ over an FS-domain $L$ is an $F S_{+}$-domain.
\end{abstract}

\section{Introduction}

Powerdomains are very important structures in Domain theory, which play an important role in modeling the semantics of nondeterministic programming languages. Three classical powerdomains are the Hoare or lower powerdomain [1], the Smyth or upper powerdomain [2], and the Plotkin or convex powerdomain [3]. They are all free dcpo-algebras over (continuous) dcpos with special binary operators satisfying some equations and inequalities (see [4-12]).

In [13], Huth et al. concluded that the Hoare powerdomain $P^{H}(L)$ over a pointed domain $L$ is a distributive $F S_{\vee}$-lattice. In [14], Meng and Kou obtained that the Smyth powerdomain $P^{S}(L)$ of a Lawson compact domain $L$ is an $F S_{\wedge}$-domain. Then we have a problem whether the Plotkin powerdomain can be characterized by some special FSdomain. In this paper, we will introduce a new domain construction called the $F S_{+}$-domain which is a +-semilattice and there exists a directed family of finitely separated Scott continuous and +-semilattice homomorphisms which can approximate $i d_{L}$, where the operation + is Scott continuous which satisfied the commutative, associative, and idempotency laws. And the category with $F_{+}$-domains as objects and Scott continuous functions as morphisms is a Cartesian closed category. We will show that the Plotkin powerdomain $P^{P}(L)$ over an FS-domain $L$ is an $F_{+}$-domain, where the Plotkin powerdomain is the free dcpo-semilattice over a continuous dcpo.

Next, we collect some basic notions needed in this paper. The reader can also consult $[4,5,15,16]$. A poset $L$ is called a directed complete poset (a dcpo, for short) if any nonempty directed subset of $L$ has a sup in $L$. For $x, y \in L, x$ is way below $y$ (denoted by $x \ll y$ ) if and only if, for all directed subsets $D \subseteq L$ for which sup $D$ exists, the relation $y \leq \sup D$ implies the existence of a $d \in D$ with $x \leq d$. A dcpo $L$ is called a continuous domain if, for all $x \in L, x=\bigvee^{\uparrow} \downarrow x$; that is, the set $\downarrow x=\{a \in L: a \ll x\}$ is directed and $x=\bigvee\{a \in L: a \ll x\}$. For a subset $A$ of $L$, let $\uparrow A=\{x \in L: \exists a \in A, a \leq x\}, \downarrow A=$ $\{x \in L: \exists a \in A, x \leq a\}$. We use $\uparrow a$ (resp., $\downarrow a$ ) instead of $\uparrow\{a\}$ (resp., $\downarrow\{a\}$ ) when $A=\{a\} . A$ is called an upper (resp., a lower) set if $A=\uparrow A$ (resp., $A=\downarrow A$ ). If $(L, \leq)$ is a dcpo, we define the Scott topology, denoted by $\sigma(L)$, which has as its topology of closed sets all directed complete lower subsets, that is, lower sets closed under directed sups. A function $f$ from a dcpo $L$ into a dcpo $P$ is continuous with respect to the Scott topologies if $f$ preserves suprema of directed subsets.

Recall the definition of FS-domain: a dcpo $L$ is called an $F S$-domain if $i d_{L}$ is approximated directly by a family of finitely separated Scott continuous functions. A Scott continuous function $f: L \rightarrow L$ is called finitely separated if there exists a finite set $M_{f}$ such that, for each $x \in L$, there exists $m \in M_{f}$ such that $f(x) \leq m \leq x$.

\section{2. $F S_{+}$-Domains}

2.1. Categories of $F S_{+}$-Domains. For dcpos $L$ and $P$, the function space $[L \rightarrow P]$ of all Scott continuous functions from $L$ to $P$ with the pointwise order is a dcpo. Then for dcpo +-semilattices $D$ and $E$, we conclude that the function 
space $\left[D \rightarrow{ }_{+} E\right]$ of all the Scott continuous and +-semilattice homomorphisms from $D$ to $E$ with the pointwise order is a dcpo +-semilattice from the following theorem, where the operation + satisfies the commutative, associative, and idempotency laws.

Theorem 1. Let $D$ and $E$ be dcpo +-semilattices; then $\left[D \rightarrow{ }_{+} E\right]$ is a dcpo +-semilattice.

Proof. For any directed family $\left\{f_{j} \in\left[D \rightarrow{ }_{+} E\right]: j \in J\right\}$ and $x \in D$, set $f(x)=\bigvee_{j \in J} f_{j}(x)$. It is obvious that $f$ is Scott continuous. Then

$$
\begin{aligned}
f(x+y) & =\bigvee_{j \in J} f_{j}(x+y)=\bigvee_{j \in J}\left(f_{j}(x)+f_{j}(y)\right) \\
& =\left(\bigvee_{j \in J} f_{j}(x)\right)+\left(\bigvee_{j \in J} f_{j}(y)\right)=f(x)+f(y) .
\end{aligned}
$$

So $f$ is also a Scott continuous and +-semilattice homomorphism. Hence $\left[D \rightarrow{ }_{+} E\right]$ is a dcpo.

For any $x \in D, f, g \in\left[D \rightarrow{ }_{+} E\right]$, we define $(f+g)(x)=$ $f(x)+g(x)$. For a directed set $\left\{x_{k} \in D: k \in K\right\}$, we have

$$
\begin{aligned}
(f+g)\left(\bigvee_{k \in K}\left(x_{k}\right)\right) & =f\left(\bigvee_{k \in K}\left(x_{k}\right)\right)+g\left(\bigvee_{k \in K}\left(x_{k}\right)\right) \\
& =\bigvee_{k \in K} f\left(x_{k}\right)+\bigvee_{k \in K} g\left(x_{k}\right) \\
& =\bigvee_{k \in K} \bigvee_{k^{\prime} \in K}\left[f\left(x_{k}\right)+g\left(x_{k^{\prime}}\right)\right] \\
& =\bigvee_{k \in K}\left[f\left(x_{k}\right)+g\left(x_{k}\right)\right] \\
& =\bigvee_{k \in K}\left[(f+g)\left(x_{k}\right)\right] .
\end{aligned}
$$

Then $f+g$ is Scott continuous.

For a pair of points $x, y$ in $D$,

$$
\begin{aligned}
(f+g)(x+y) & =f(x+y)+g(x+y) \\
& =(f(x)+f(y))+(g(x)+g(y)) \\
& =(f(x)+g(x))+(f(y)+g(y)) \\
& =(f+g)(x)+(f+g)(y) .
\end{aligned}
$$

That is, $f+g$ is a + -semilattice homomorphism. So $\left[D \rightarrow{ }_{+} E\right]$ is a +-semilattice.

Finally, by the Scott continuity of the operation + , we obtain the following conclusion. For the sup of the directed set $\left\{f_{j} \in\left[D \rightarrow{ }_{+} E\right]: j \in J\right\}$ and $g \in\left[D \rightarrow{ }_{+} E\right]$, if $x \in D$, then

$$
\begin{aligned}
{\left[g+\left(\bigvee_{j \in J} f_{j}\right)\right](x) } & =g(x)+\left(\bigvee_{j \in J} f_{j}(x)\right) \\
& =\bigvee_{j \in J}\left[g(x)+f_{j}(x)\right] \\
& =\bigvee_{j \in J}\left[\left(g+f_{j}\right)(x)\right] \\
& =\left[\bigvee_{j \in J}\left(g+f_{j}\right)\right](x) .
\end{aligned}
$$
tinuous.

So $+:\left[D \rightarrow{ }_{+} E\right] \times\left[D \rightarrow{ }_{+} E\right] \rightarrow\left[D \rightarrow{ }_{+} E\right]$ is Scott con-

We have obtained that $\left[D \rightarrow{ }_{+} E\right]$ is a dcpo +-semilattice.

With respect to these special Scott continuous functions, we will introduce some new order structures.

Definition 2. A dcpo $L$ is called an $F S_{+}$-domain if it is a +semilattice and there exists a directed family of finitely separated Scott continuous and +-semilattice homomorphisms which can approximate $i d_{L}$.

For example, an $F S_{\wedge}$-domain is a continuous dcpo $\wedge$ semilattice where id is approximated by a directed family of finitely separated Scott continuous functions preserving finite infs.

We know that an $F S_{+}$-domain is an FS-domain.

Theorem 3. Let $D$ and $E$ be $F S_{+}$-domains; then $\left[D \rightarrow{ }_{+} E\right]$ and $[D \rightarrow E]$ are $\mathrm{FS}_{+}$-domains.

Proof. Suppose that $\mathscr{D}$ and $\mathscr{E}$ are approximate identities for $D$ and $E$, respectively. Then we claim that the family

$$
\mathscr{D} \otimes \mathscr{E}=\{\delta \otimes \epsilon: \delta \in \mathscr{D}, \epsilon \in \mathscr{E}\},
$$

defined by

$$
f \longmapsto \epsilon^{2} f \delta^{2}
$$

for $f \in\left[D \rightarrow{ }_{+} E\right]$ is an approximate identity for $\left[D \rightarrow{ }_{+} E\right]$ where $\delta \otimes \epsilon$ is finitely separated. The proof is similar to the case of FS-domains.

It suffices to show that $\delta \otimes \epsilon \in\left[D \rightarrow{ }_{+} E\right] \rightarrow_{+}\left[D \rightarrow{ }_{+} E\right]$. Firstly, it is obvious that $\delta \otimes \epsilon$ is Scott continuous. Secondly, for a pair of points $f, g \in\left[D \rightarrow{ }_{+} E\right]$, we have for any $x \in D$

$$
\begin{aligned}
{[(\delta \otimes \epsilon)(f+g)](x) } & =\left[\epsilon^{2}(f+g) \delta^{2}\right](x) \\
& =\epsilon^{2}\left[f \delta^{2}(x)+g \delta^{2}(x)\right]
\end{aligned}
$$




$$
\begin{aligned}
& =\epsilon\left[\epsilon\left(f \delta^{2}(x)+g \delta^{2}(x)\right)\right] \\
& =\epsilon^{2} f \delta^{2}(x)+\epsilon^{2} g \delta^{2}(x) \\
& =\left[\epsilon^{2} f \delta^{2}+\epsilon^{2} g \delta^{2}\right](x) \\
& =[(\delta \otimes \epsilon)(f)+(\delta \otimes \epsilon)(g)](x) .
\end{aligned}
$$

So we conclude that $\delta \otimes \epsilon$ is a + -semilattice homomorphism. Then $\left[D \rightarrow{ }_{+} E\right]$ is an $F S_{+}$-domain. Similarly, $[D \rightarrow$ $E]$ is also an $F S_{+}$-domain.

Theorem 4. The category with $\mathrm{FS}_{+}$-domains as objects and Scott continuous functions as morphisms is a Cartesian closed category.

Note that the category with $F S_{+}$-domains as objects and Scott continuous and +-semilattice homomorphisms as morphisms is not a Cartesian closed category generally, because the evaluation maps do not preserve the finite +operation.

\subsection{Classify the Powerdomains}

Definition 5 (see [5]). Let $L$ be a dcpo-algebra equipped with a Scott continuous binary operation + that satisfies the following equations: for any $a, b, c, \in L$

(1) $a+a=a$ (idempotency law);

(2) $a+b=b+a$ (commutative law);

(3) $a+(b+c)=(a+b)+c$ (associative law).

Then the dcpo-algebra is a commutative idempotent semigroup, called a dcpo-semilattice. The free dcpo-semilattice over a dcpo $L$ is called the convex or Plotkin powerdomain of $L$ and it is denoted by $P^{P}(L)$.

If the binary operation + satisfies the inequality $a+b \leq a$, then we obtain the upper or Smyth powerdomain, and it is denoted by $P^{S}(L)$, where $a+b=a \wedge b$.

Similarly, if the binary operation + satisfies $a+b \geq a$, then it is called the lower or Hoare powerdomain, denoted by $P^{H}(L)$, where $a+b=a \vee b$.

Proposition 6 (see [5]). For subsets $C$ and $D$ of a preordered set $(L, \leq)$ one has

(1) $C={ }_{H} \downarrow C$;

(2) $C \leq_{H} D$ iff $\downarrow C \subseteq \downarrow D$;

(3) $C \ll_{H} D$ iff there exists a finite subset $F \subseteq L$ such that $C \subseteq \downarrow F \subseteq \downarrow D ;$

(4) $C={ }_{S} \uparrow C$;

(5) $C \leq_{S} D$ iff $\uparrow D \subseteq \uparrow C$;

(6) $C \ll_{S} D$ iff $D \subseteq$ int $_{\sigma}(\uparrow C)$ iff $D \subseteq \uparrow C$;

(7) $C={ }_{P} \downarrow C \cap \uparrow C=\sup \left\{\downarrow F \cap \uparrow F: F \prec C, F \subseteq_{\text {fin }} L\right\}$, where $F \prec C$ iff $F \subseteq \downarrow C$ and $C \subseteq \uparrow F$;

(8) $C \leq_{P} D$ iff $\downarrow C \subseteq \downarrow D$ and $\uparrow D \subseteq \uparrow C$;
(9) $C \ll_{P} D$ iff $C \ll_{H} D$ and $C \ll_{S} D$.

Next, we draw the conclusion that some special FSdomain categories concerning the operation + can be used to classify the powerdomains.

Theorem 7. If $L$ is an FS-domain, then the convex powerdomain $P^{P}(L)$ is an $\mathrm{FS}_{+}$-domain.

Proof. Suppose that $L$ is an FS-domain; then $L$ is a Lawson compact domain. Thus, $P^{P}(L)$ is also a domain. Assume that $\mathscr{F}=\left\{f_{i}: L \rightarrow L\right\}_{i \in I}$ is the approximate identity for $L$, where $\mathscr{F}$ is a family of finitely separated Scott continuous functions; that is, for any $f_{i}$, there exists a finite set $M_{i} \subseteq L$ such that, for any $x \in L$, there exists some $m \in M_{i}$ such that $f_{i}(x) \leq m \leq x$. We claim that $\left\{P\left(f_{i}\right): P^{P}(L) \rightarrow P^{P}(L)\right\}_{i \in I}$ is the approximate identity for $P^{P}(L)$. It suffices to consider four steps as follows.

(1) $P\left(f_{i}\right) \leq P(i d)$. For $A \in P^{P}(L)$, define $P\left(f_{i}\right)(A)=$ $P\left(f_{i}(A)\right)=\downarrow \quad f_{i}(A) \cap \uparrow f_{i}(A)$. By Proposition 6, $\downarrow f_{i}(A) \cap \uparrow f_{i}(A) \in P^{P}(L)$. For any $x \in A$, let $M_{i}(A)=\left\{m \in M_{i}: \exists x \in A, f_{i}(x) \leq m \leq x\right\}$; then $f_{i}(x) \leq x$ implies $\downarrow f_{i}(A) \subseteq \downarrow M_{i}(A) \subseteq \downarrow A$ and $\uparrow A \subseteq$ $\uparrow M_{i}(A) \subseteq \uparrow f_{i}(A)$. Hence $P\left(f_{i}\right)(A)=P\left(f_{i}(A)\right) \leq_{P} A$.

(2) $\sup \left\{P\left(f_{i}\right): i \in I\right\}=P(i d)$. For any $A \in P^{P}(L)$, it is obvious that $\sup \left\{P\left(f_{i}\right)(A): i \in I\right\} \leq A$. Suppose $A \not$ $\sup \left\{P\left(f_{i}\right)(A): i \in I\right\}$. There is $B \in P^{P}(L)$ such that $B \ll_{P} A$ and $B \not \sup \left\{P\left(f_{i}\right)(A): i \in I\right\}$. By $B \ll_{P} A$ and $A=\sup \left\{\downarrow F \cap \uparrow F: F \prec A, F \subseteq \subseteq_{f i n} L\right\}$, there is some finite set $F \prec A$ such that $B \leq \downarrow F \cap \uparrow F$. But for any finite set $F \prec A$, we have $F=\sup \left\{f_{i}(F)\right.$ : $i \in I\}$, where $F \prec A$ iff $F \subseteq \downarrow A$ and $A \subseteq \uparrow F$. Then $\downarrow F \cap \uparrow F=\sup \left\{\downarrow f_{i}(F) \cap \uparrow f_{i}(F): i \in I\right\} \leq \sup \{\downarrow$ $\left.f_{i}(A) \cap \uparrow f_{i}(A): i \in I\right\}$. This is a contradiction. Then we conclude that $\sup \left\{P\left(f_{i}\right): i \in I\right\}=P(i d)$.

(3) $P\left(f_{i}\right)$ is Scott continuous and finitely separated. For a directed family $\mathscr{D}$ in $P^{P}(L)$, we have

$$
\begin{aligned}
P\left(f_{i}\right)(\sup \mathscr{D}) & =P\left(f_{i}(\sup \mathscr{D})\right) \\
& =P\left(\sup \left\{f_{i}(D): D \in \mathscr{D}\right\}\right) \\
& =\sup \left\{P\left(f_{i}(D)\right): D \in \mathscr{D}\right\} .
\end{aligned}
$$

Then $P\left(f_{i}\right)$ is Scott continuous. For any $A \in P^{P}(L)$, $M_{i}(A)$ is a finite set. By $\downarrow f_{i}(A) \subseteq \downarrow M_{i}(A) \subseteq \downarrow A$ and $\uparrow A \subseteq \uparrow M_{i}(A) \subseteq \uparrow f_{i}(A)$, it follows that $\downarrow M_{i}(A) \cap \uparrow$ $M_{i}(A) \in P^{P}(L)$. Let $\mathscr{M}_{i}=\left\{\downarrow M_{i}(A) \cap \uparrow M_{i}(A): A \epsilon\right.$ $\left.P^{P}(L)\right\}$. Since $M_{i}(A) \subseteq M_{i}$ and $M_{i}$ is finite, it follows that $\mathscr{M}_{i}$ is a finite family of $P^{P}(L)$. And we have that, for any $A$, there exists $\downarrow M_{i}(A) \cap \uparrow M_{i}(A) \in \mathscr{M}_{i}$ such that $P\left(f_{i}\right)(A) \leq_{P} \downarrow M_{i}(A) \cap \uparrow M_{i}(A) \leq_{P} A$; that is, $P\left(f_{i}\right)$ is finitely separated. 
(4) $P\left(f_{i}\right)$ is a +-semilattice homomorphism. For $A, B \in$ $P^{P}(L)$, since $P^{P}(L)$ is a +-semilattice, $A+B=\downarrow(A \cup$ $B) \cap \uparrow(A \cup B) \in P^{P}(L)$ :

$$
\begin{aligned}
P\left(f_{i}\right)(A+B)= & P\left(f_{i}\right)[\downarrow(A \cup B) \cap \uparrow(A \cup B)] \\
= & \downarrow f_{i}[\downarrow(A \cup B) \cap \uparrow(A \cup B)] \\
& \cap \uparrow f_{i}[\downarrow(A \cup B) \cap \uparrow(A \cup B)] \\
= & \downarrow f_{i}(A \cup B) \cap \uparrow f_{i}(A \cup B) \\
= & \downarrow\left[\left(\downarrow f_{i}(A) \cap \uparrow f_{i}(A)\right)\right. \\
& \left.\cup\left(\downarrow f_{i}(B) \cap \uparrow f_{i}(B)\right)\right] \\
& \cap \uparrow\left[\left(\downarrow f_{i}(A) \cap \uparrow f_{i}(A)\right)\right. \\
\left.\cup\left(\downarrow f_{i}(B) \cap \uparrow f_{i}(B)\right)\right] & P\left(f_{i}\right)(A)+P\left(f_{i}\right)(B) .
\end{aligned}
$$

Then we conclude that $\left\{P\left(f_{i}\right): i \in I\right\}$ is the approximate identity for $P^{P}(L)$. Thus the convex powerdomain $P^{P}(L)$ is an $F S_{+}$-domain.

Combined with the work of Huth et al. [13] and Meng and Kou [14], we conclude the following theorem.

Theorem 8. Let L be a domain. Then the following statements hold:

(1) if $L$ is Lawson compact, then the Smyth powerdomain $P^{S}(L)$ is an $F S_{\Lambda^{-d o m a i n}}$ (in [14]);

(2) if $L$ has a least point, then the Hoare powerdomain

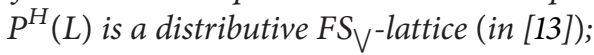

(3) if $L$ is an FS-domain, then the Plotkin powerdomain $P^{P}(L)$ is an $F_{+}$-domain.

\section{Conflict of Interests}

The authors declare that there is no conflict of interests regarding the publication of this paper.

\section{Acknowledgments}

This work is supported by the Foundation of the Education Department of Henan Province (13A110552), the Foundation of the Science and Technology Department of Henan Province (142300410165), and the Foundation of Henan Normal University (2013PL03).

\section{References}

[1] M. B. Smyth, "Power domains and predicate transformers: a topological view," in Automata, Languages and Programming, vol. 154 of Lecture Notes in Computer Science, pp. 662-675, Springer, Berlin, Germany, 1983.
[2] M. B. Smyth, "Powerdomains," Journal of Computer and Systems Sciences, vol. 16, pp. 23-36, 1978.

[3] G. D. Plotkin, "A powerdomain construction," SIAM Journal on Computing, vol. 5, no. 3, pp. 452-487, 1976.

[4] S. Abramsky and A. Jung, "Domain theory," in Handbook of Logic in Computer Science, vol. 3, Oxford University Press, New York, NY, USA, 1994.

[5] G. Gierz, K. H. Hofmann, K. Keimel, J. D. Lawson, M. Mislove, and D. S. Scott, Continuous lattices and domains, vol. 93 of Encyclopedia of Mathematics and its Applications, Cambridge University Press, New York, NY, USA, 2003.

[6] R. Heckmann and K. Keimel, "Quasicontinuous domains and the Smyth powerdomain," Electronic Notes in Theoretical Computer Science, vol. 298, pp. 215-232, 2013.

[7] R. Heckmann, Power domain constructions [Ph.D. thesis], Universität des Saarlandes, Saarbrücken, Germany, 1990.

[8] R. Heckmann, "An upper power domain construction in terms of strongly compact sets," in Mathematical Foundations of Programming Semantics, vol. 598 of Lecture Notes in Computer Science., pp. 272-293, Springer, Berlin, Germany, 1992.

[9] R. Heckmann, "Stable power domains," Theoretical Computer Science, vol. 136, no. 1, pp. 21-56, 1994.

[10] R. Heckmann, "Characterising FS domains by means of power domains," Theoretical Computer Science, vol. 264, no. 2, pp. 195203, 2001.

[11] J. H. Liang and H. Kou, "Convex power domain and Vietoris space," Computers \& Mathematics with Applications, vol. 47, no. 4-5, pp. 541-548, 2004.

[12] M. Mislove, "On the Smyth power domain," in Mathematical Foundations of Programming Language Semantics, vol. 298 of Lecture Notes in Computer Science, pp. 161-172, Springer, Berlin, Germany, 1988.

[13] M. Huth, A. Jung, and K. Keimel, "Linear types, approximation, and topology," in Proceedings of the IEEE 9th Annual Symposium on Logic in Computer Science, pp. 110-114, July 1994.

[14] H. Meng and H. Kou, "Function spaces of semilattice homomorphisms and $F S_{\wedge}$-domains," Chinese Annals of Mathematics A, vol. 32, no. 1, pp. 107-114, 2011.

[15] A. Jung, Cartesian Closed Categories of Domains, vol. 66 of CWI Tracts, Stichting Mathematisch Centrum Centrum voor Wiskunde en Informatica, Amsterdam, The Netherlands, 1989.

[16] J. D. Lawson, “The versatile continuous order," in Mathematical Foundations of Programming Language Semantics, vol. 298 of Lecture Notes in Computer Science, pp. 134-160, Springer, Berlin, Germany, 1988. 


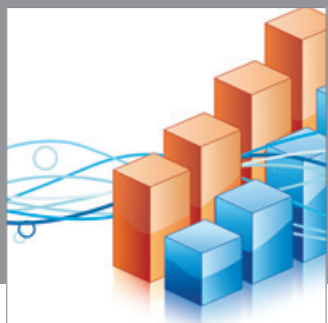

Advances in

Operations Research

mansans

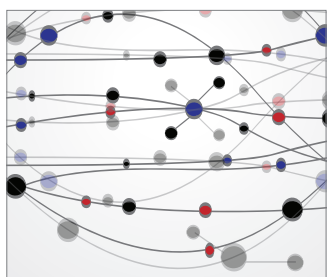

The Scientific World Journal
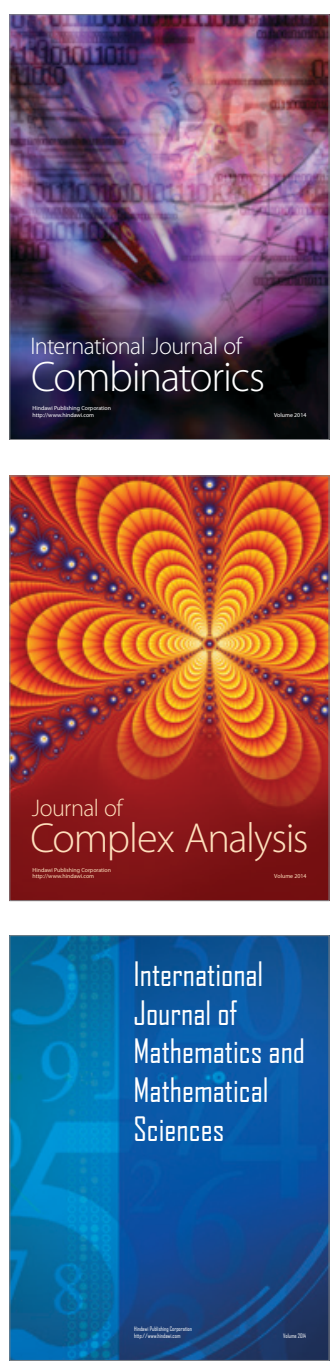
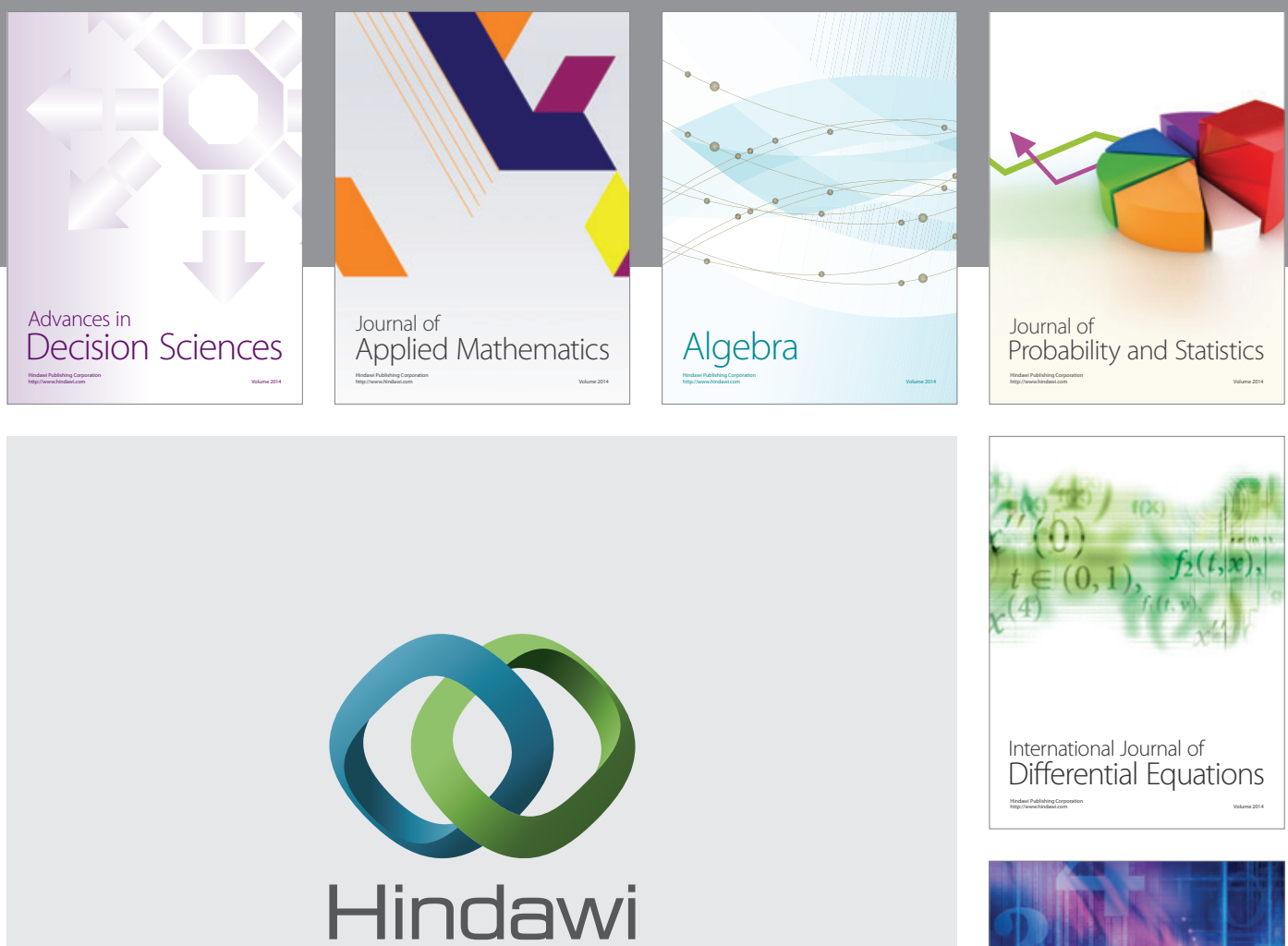

Submit your manuscripts at http://www.hindawi.com
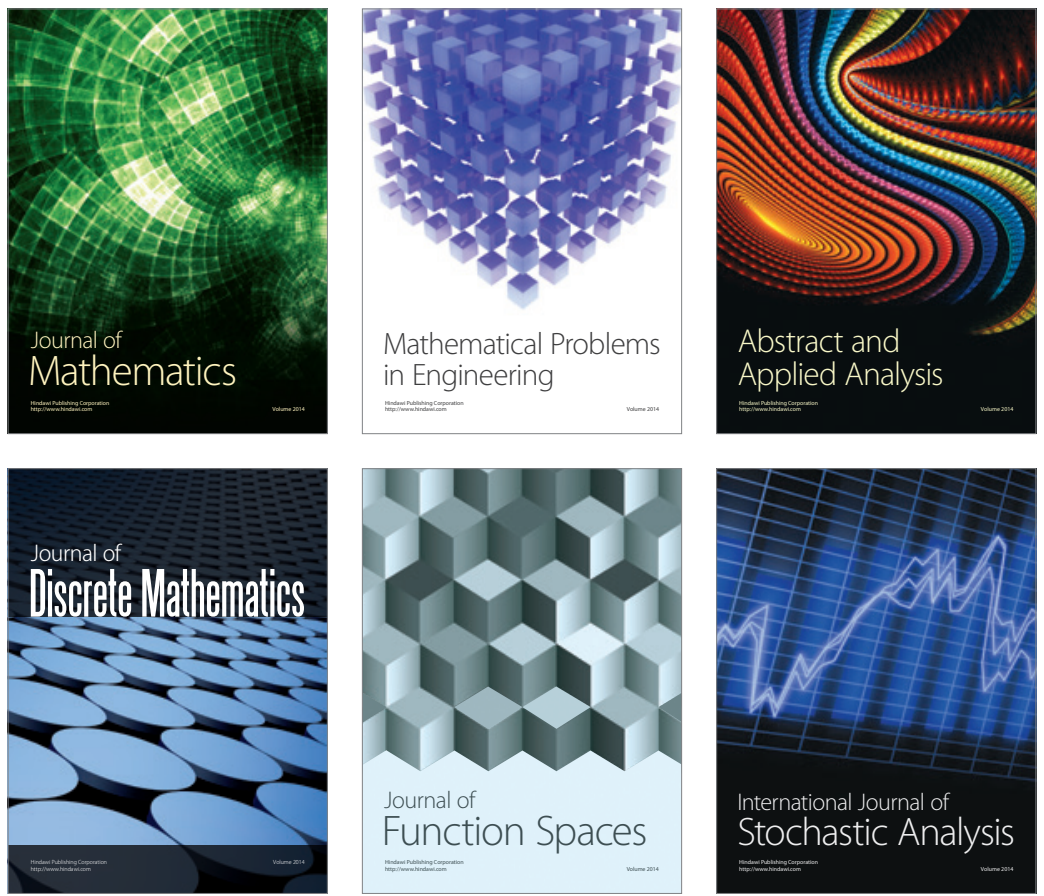

Journal of

Function Spaces

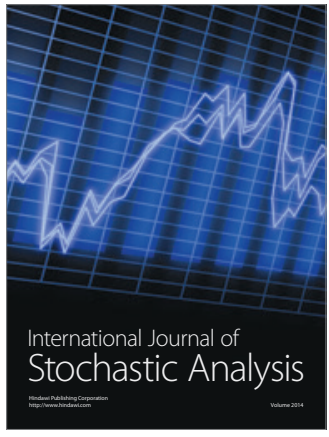

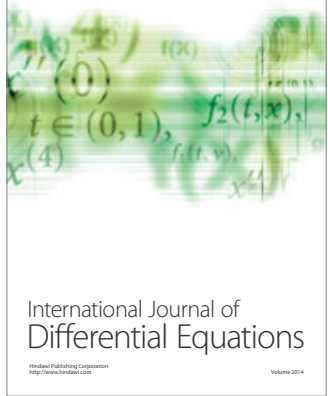
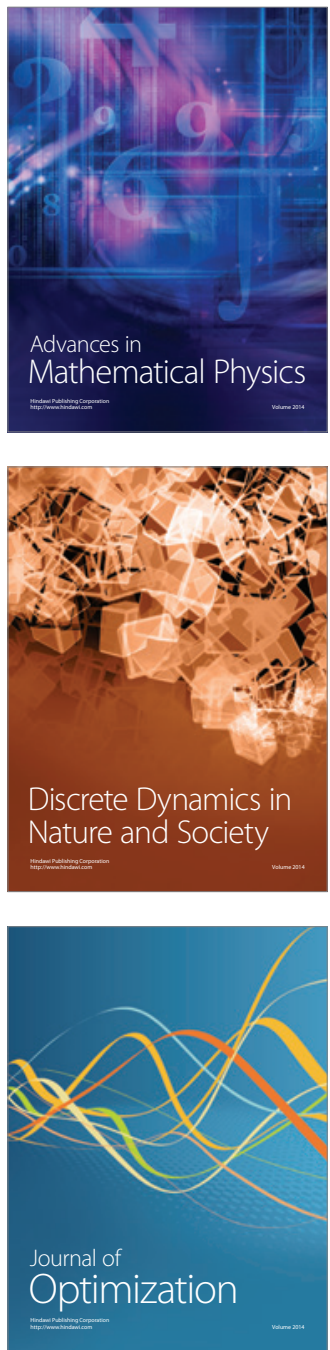